\title{
Biomechanical Study of Posterior Pelvic Fixations in Vertically Unstable Sacral Fractures: An Alternative to Triangular Osteosynthesis
}

\author{
Weera Chaiyamongkol, Apipop Kritsaneephaiboon, Piyawat Bintachitt, \\ Sitthiphong Suwannaphisit, Boonsin Tangtrakulwanich \\ Department of Orthopaedic Surgery and Physical Medicine, Faculty of Medicine, Prince of Songkla University, Songkhla, Thailand
}

\begin{abstract}
Study Design: Biomechanical study.
Purpose: To investigate the relative stiffness of a new posterior pelvic fixation for unstable vertical fractures of the sacrum.

Overview of Literature: The reported operative fixation techniques for vertical sacral fractures include iliosacral screw, sacral bar fixations, transiliac plating, and local plate osteosynthesis. Clinical as well as biomechanical studies have demonstrated that these conventional techniques are insufficient to stabilize the vertically unstable sacral fractures.

Methods: To simulate a vertically unstable fractured sacrum, 12 synthetic pelvic models were prepared. In each model, a 5-mm gap was created through the left transforaminal zone (Denis zone II). The pubic symphysis was completely separated and then stabilized using a 3.5-mm reconstruction plate. Four each of the unstable pelvic models were then fixed with two iliosacral screws, a tension band plate, or a transiliac fixation plus one iliosacral screw. The left hemipelvis of these specimens was docked to a rigid base plate and loaded on an S1 endplate by using the Zwick Roell z010 material testing machine. Then, the vertical displacement and coronal tilt of the right hemipelves and the applied force were measured.

Results: The transiliac fixation plus one iliosacral screw constructions could withstand a force at $5 \mathrm{~mm}$ of vertical displacement greater than the two iliosacral screw constructions $(p=0.012)$ and the tension band plate constructions $(p=0.003)$. The tension band plate constructions could withstand a force at $5^{\circ}$ of coronal tilt less than the two iliosacral screw constructions $(p=0.027)$ and the transiliac fixation plus one iliosacral screw constructions $(p=0.049)$.

Conclusions: This study proposes the use of transiliac fixation in addition to an iliosacral screw to stabilize vertically unstable sacral fractures. Our biomechanical data demonstrated the superiority of adding transiliac fixation to withstand vertical displacement forces.
\end{abstract}

Keywords: Sacrum; Fracture fixation; Biomechanics; lliosacral screw; Triangular osteosynthesis

\section{Introduction}

Operative treatment is often required for unstable sacral fractures. Treating unstable fracture located in the poste- rior pelvic ring requires consideration of the stability of the region into account. An unstable sacral fracture with posterior pelvic ring disruptions can result in rotational and vertical instability of the pelvis. The current operative

Received Jan 25, 2018; Revised Apr 10, 2018; Accepted May 22, 2018

Corresponding author: Weera Chaiyamongkol

Department of Orthopaedic Surgery and Physical Medicine, Faculty of Medicine, Prince of Songkla University, Hat Yai, Songkhla 90110, Thailand

Tel: +66-7445-1601, Fax: +66-7442-9898, E-mail: weera_c@yahoo.com 


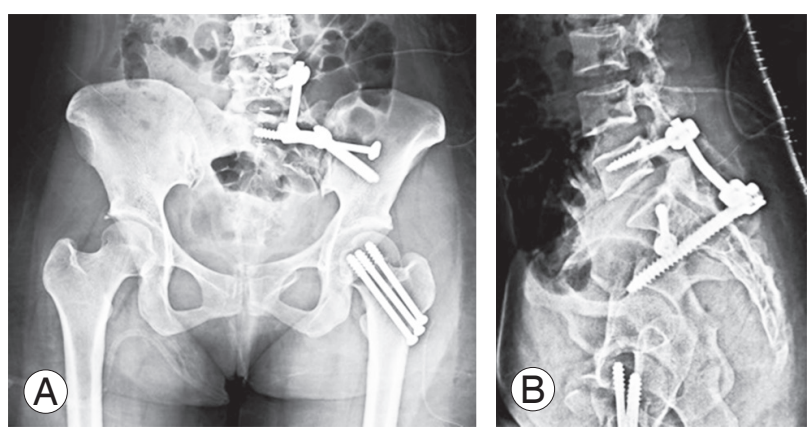

Fig. 1. (A, B) Radiographs show a vertically unstable fracture of the sacrum treated with triangular osteosynthesis.

techniques include iliosacral screw, sacral bar fixations, transiliac plating, and local plate osteosynthesis [1-4]. However, clinical and biomechanical studies have demonstrated that these conventional techniques are often insufficient to stabilize vertically unstable sacral fractures [5-8].

Kach and Trentz [9] described a technique called as distraction spondylodesis to counter the vertical shear forces existing in vertically unstable sacral fracture zones II and III (Denis) [10]. This technique involves a transfixation from L5 to the pelvic rim without any direct fixation across the fracture site. In another study, Schildhauer et al. [11] introduced the use of triangular osteosynthesis. This fixation was a variation of the technique described earlier by Kach and Trentz [9]. Schildhauer et al. [11] used a supplemental iliosacral screw to maintain sacral fracture reduction (Fig. 1). Another biomechanical study concluded that triangular osteosynthesis was superior to iliosacral screw fixation [7]. Patients with former fixation are allowed to undertake early progressive weight-bearing activities $[11,12]$. However, the drawback of this technique is the need for fixation across the L5 to S1 or even L4 to S1 motion segments, thereby leading to loss of motion and/ or possible chronic back pain $[13,14]$.

As a potentially superior option to triangular osteosynthesis, we devised a technique involving transiliac fixation with iliosacral screw fixation for vertically unstable sacral fractures. The transiliac fixation could reinforce the iliosacral screw without touching the lumbosacral motion segment. The purpose of this study was to ascertain biomechanical advantage of adding a transiliac fixation in case of vertically unstable sacral fractures.

\section{Materials and Methods}

This work was a biomechanical investigation of synthetic
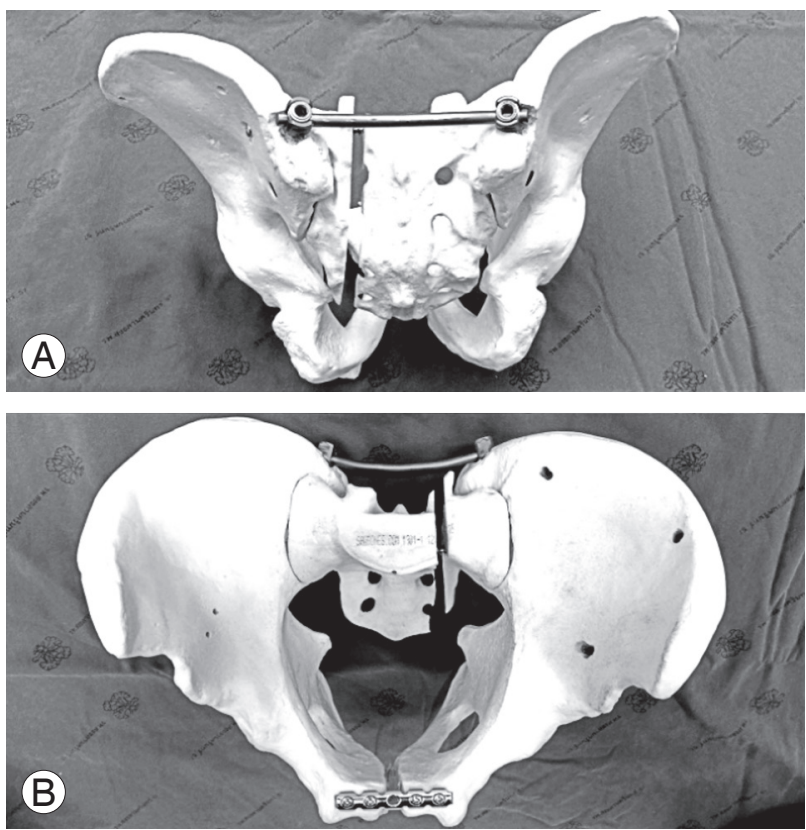

Fig. 2. (A, B) Posterior stabilization in an unstable synthetic pelvis consisting of (1) one iliosacral screw, fully threaded $(6.5-\mathrm{mm}$ diameter $\times 90-\mathrm{mm}$ length) and (2) transiliac fixation consisting of two standard mono-axial pedicle screws (8.5-mm diameter $\times 100-\mathrm{mm}$ length) connected with a titanium rod (Ti-6AI-4V ELI, ASTM F136).

pelvic models. After obtaining approval from the institutional review board of Human Research Ethics Committee, Faculty of Medicine, Prince of Songkla University (IRB approval no., REC 58-057-11-1), 12 synthetic pelvic models (Pacific Research Laboratories Inc., Vashon, WA, USA) were prepared to simulate the vertically unstable fractured sacrum. In each model, a 5-mm gap was created through the left transforaminal zone (Denis zone II). The pubic symphysis was completely separated and then stabilized using a $3.5-\mathrm{mm}$ reconstruction plate. Four each of the unstable pelvic models were fixed with (1) two iliosacral screws, (2) a tension band plate, or (3) a transiliac fixation plus one iliosacral screw (Fig. 2). A fully threaded cancellous screw (6.5 $\mathrm{mm}$ in diameter, $90 \mathrm{~mm}$ in length) was employed as the iliosacral screw. An appropriately sized 15-hole reconstruction plate with $3.5-\mathrm{mm}$ cortical screws was used as the posterior tension band plate. Transiliac fixation consisted of one $8.0 \times 100-\mathrm{mm}$ monoaxial pedicle screw inserted into each ilium at the posterior superior iliac spine. The screw was inserted through the dense bone above the superior aspect of the sciatic notch, and the screws present in both the ilia were connected using a $6.0-\mathrm{mm}$ rod. To simulate a single-leg stance model, the 
left hemipelvis of all the models was docked to a rigid base plate modified from the Moore's prosthesis. Distance (HC-SR04) and gyro (UM7 Orientation Sensor) sensors were then installed into the right hemipelvis to detect the vertical displacement and coronal tilt, respectively. Each specimen was vertically loaded onto an S1 endplate by using the Zwick Roell z010 material testing machine (Zwick, Ulm, Germany) (Fig. 3). Prior testing with a static load of $10 \mathrm{~mm} / \mathrm{min}$ on each pelvic model demonstrated that the two iliosacral screw constructions, tension band plate construction, and transiliac fixation plus one iliosacral screw construction could withstand a maximum vertical load of $280 \mathrm{~N}, 161 \mathrm{~N}$, and $510 \mathrm{~N}$, respectively. Therefore, the loading sequences were started with a cyclical preload

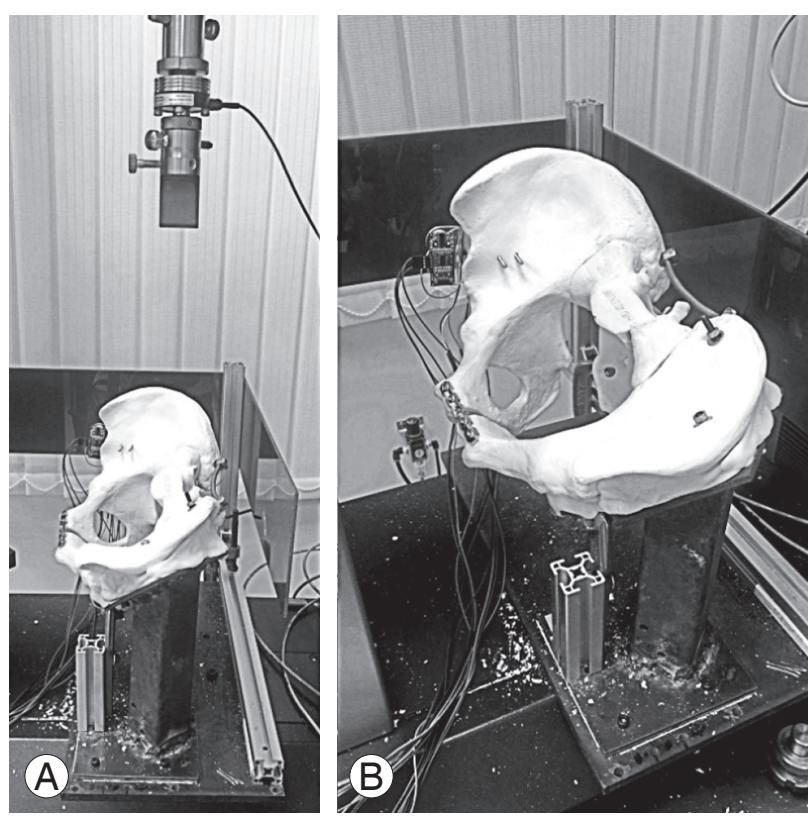

Fig. 3. (A, B) Photographs showing our experimental setup with a synthetic bone in the loading frame. The left hemipelvis was stabilized in a rigid base plate to simulate a single limb stance model.
( 25 cycles, $120 \mathrm{~mm} / \mathrm{min}$ ) up to $30 \%$ of the maximum withstandable force in each construction type, followed by a subsequent static load of $10 \mathrm{~mm} / \mathrm{min}$. During the loading process, vertical displacement and the coronal tilt of the right hemipelvis along with applied force were measured.

The normality of the data was confirmed with the Shapiro-Wilk and Kolmogorov-Smirnov tests. One-way analysis of variance was used to determine the force for displacement between the pelvic constructions. Post hoc test was applied for multiple comparisons. All statistical analyses were conducted using SPSS ver. 17.0 (SPSS Inc., Chicago, IL, USA). A $p<0.05$ was considered statistically significant.

\section{Results}

\section{Vertical displacement}

The mean values of force $(\mathrm{N})$ required for 5-mm vertical displacement of the right hemipelves are shown in Table 1. The transiliac fixation plus one iliosacral screw construction could withstand a force (mean, 286.8 \pm 53.2 ) at $5 \mathrm{~mm}$ of vertical displacement greater than the two iliosacral screws $(p=0.012)$ and the tension band plate $(p=0.003)$ constructions. No statistically significant differences were observed in the force required for 5 -mm vertical displace-

Table 1. Force (N) at $5 \mathrm{~mm}$ vertical displacement

\begin{tabular}{lr} 
Fixation type & Mean \pm standard deviation \\
\hline Two ISS & $152.51 \pm 42.48$ \\
\hline Tension band plate & $118.42 \pm 57.13$ \\
\hline Transiliac fixation+one ISS & $286.82 \pm 53.21$ \\
\hline
\end{tabular}

ISS, iliosacral screw.

Table 2. Multiple comparisons of force (N) for $5 \mathrm{~mm}$ vertical displacement

\begin{tabular}{llcc} 
Fixation type (1) & \multicolumn{1}{c}{ Fixation type (2) } & Mean difference (1)-(2) & $p$-value \\
\hline Two ISS & Tension band plate & 34.08 & 0.631 \\
& Transiliac fixation+one ISS & -134.31 & $0.012^{*}$ \\
\hline Tension band plate & Two ISS & -34.08 & 0.631 \\
\hline Transiliac fixation+one ISS & Transiliac fixation+one ISS & -168.39 & $0.003^{*}$ \\
\hline
\end{tabular}

ISS, iliosacral screw.

*Statistically significant if $p<0.05$. 
ment between the two iliosacral screws and the tension band plate $(p=0.631)$ constructions (Table 2$)$.

\section{Coronal angulation}

The mean values of force $(\mathrm{N})$ required for $5^{\circ}$ of coronal tilt of the right hemipelves are listed in Table 3. The tension band plate construction could withstand only a lesser force at $5^{\circ}$ of coronal tilt than the two iliosacral screws ( $p=0.027)$ and the transiliac fixation plus one iliosacral screw ( $p=0.049$ ) constructions. The forces required to achieve $5^{\circ}$ of coronal tilt were not significantly different between the two iliosacral screws and the transiliac fixation plus one iliosacral screw $(p=0.926)$ constructions (Table 4).

\section{Discussion}

The sacrum is unique in terms of its anatomical and mechanical aspects [15-17], and fractures in this region need specific attention. Anatomical restrictions for instrumental fixation, neural tissue proximity, and high axial load bearing pose difficulties in fracture fixation $[15,18,19]$. The iliosacral screw is one of the conventional methods for treating a vertical fracture in the sacrum. Griffin et al. [6] reported $13 \%$ failure rate with the use of a percutaneous iliosacral screw in treating unstable pelvic fractures.

Table 3. Force (N) at $5^{\circ}$ coronal tilt

\begin{tabular}{lc} 
Fixation type & Mean \pm standard deviation \\
Two ISS & $761.02 \pm 290.53$ \\
Tension band plate & $329.91 \pm 108.98$ \\
\hline Transiliac fixation+one ISS & $710.11 \pm 117.62$ \\
\hline
\end{tabular}

ISS, iliosacral screw.
The researchers established that the failures were not associated with any specific surgical techniques, rather only with the presence of a vertical sacral fracture. Keating et al. [5] analyzed the outcomes of vertically unstable pelvises treated with iliosacral screw fixation. The study reported a malunion rate of $56 \%$ and suggested augmentation with a rigid anterior fixation to minimize this occurrence. Triangular osteosynthesis, a combination of iliosacral fixation and ipsilateral lumbopelvic fixation, was originally introduced by Schildhauer et al. [11]. The problem addressed in this research was the rotational and vertical instability resulting from vertically unstable sacrum fractures. Thus, to achieve adequate stability, both transverse (iliosacral) and vertical (lumbopelvic) fixations are required to control rotational and vertical instability, respectively. One biomechanical study ascertained that an additional unilateral lumbopelvic fixation provided better stability than iliosacral screw fixation alone [7]. This result implies that lumbopelvic fixation can protect iliosacral screw and reduce the failure rate when treating patients with vertically unstable sacral fractures $[6,20]$. However, triangular osteosynthesis comes with certain disadvantages. Sagi et al. $[13,14]$ reported drawbacks including delayed union, non-union, and lumbosacral scoliosis with chronic low back pain.

As the sacrum is a part of the posterior pelvic ring and the intercalary bone between the spine and the pelvis, a fracture may cause spinopelvic dissociation wherein there is a disconnection between the cephalad lumbar spine/ central sacrum and the caudad bilateral sacral alae [21,22]. In this situation, a spinopelvic fixation, which is a bilateral lumbopelvic fixation without or with iliosacral fixation, is required to transmit the load from the spine to the ilium bilaterally $[22,23]$. In cases of spinopelvic dissociation, it is reasonable to perform a fixation at the expense of lower

Table 4. Multiple comparisons of force (N) for $5^{\circ}$ coronal tilt

\begin{tabular}{llrr} 
Fixation type (1) & Fixation type (2) & Mean difference (1)-(2) & $p$-value \\
\hline Two ISS & Tension band plate & 431.11 & $0.027^{*}$ \\
& Transiliac fixation+one ISS & 50.90 & 0.926 \\
\hline Tension band plate & Two ISS & -431.11 & $0.027^{*}$ \\
\hline Transiliac fixation+one ISS & Transiliac fixation+one ISS & -380.20 & $0.049^{*}$ \\
\hline
\end{tabular}

ISS, iliosacral screw.

*Statistically significant if $p<0.05$ 
lumbar motion. However, in cases of vertical sacral fracture without spinopelvic dissociation, iliosacral fixation plus ipsilateral lumbopelvic fixation (triangular osteosynthesis) appears to be unwarranted. Thus, instead of resorting to triangular osteosynthesis in these cases, we have suggested the use of a transiliac fixation for enhancing the iliosacral screw fixation.

The issues regarding whether cadaver or synthetic bone is more suitable for biomechanical testing remains controversial. The cadaveric pelvis is closer to a normal human pelvis; the inter-specimen variation is a concern. Furthermore, the mechanical properties of new generation synthetic bones are comparable to those of human specimens $[24,25]$. Our biomechanical study on synthetic pelvic models revealed the superiority of adding transiliac fixation for resisting vertical displacement over the use of iliosacral screw fixation alone (Table 2). Moreover, we observed that transiliac fixation was stiffer than iliosacral screw fixation alone, and hence, capable of resisting coronal tilt (Table 4). These data may encourage the usage of a transiliac fixation to supplement an iliosacral screw in case of vertically unstable sacral fractures, leaving the lumbar spine untouched.

This study, however, has several limitations. We did not compare the transiliac fixation construction with triangular osteosynthesis directly for two reasons. First, a synthetic pelvic model incorporating the lumbar spine was not available. Second, we did not consider the transverse (transiliac) fixation to be stronger than the vertical (triangular osteosynthesis) fixation in terms of vertical stability. However, we did not aim for the strongest fixation; we aimed for a strong enough fixation that leaves the lumbar spine undisturbed. Furthermore, this work was a biomechanical study involving synthetic bone; hence, we did not include the reinforcement from the soft tissues. Moreover, the research may not be able to predict the adverse outcomes in clinical situations, such as hardware prominence, nonunion, and future adverse radiographic sequelae. The ability of the transiliac fixation to supplant the triangular osteosynthesis requires further clinical studies.

\section{Conclusions}

We have proposed the use of a transiliac fixation to supplement the iliosacral screw. This approach should minimize the failure rate of iliosacral screws in treating vertically unstable sacral fractures. We believe that the proposed transiliac fixation plus iliosacral screw construction can serve as a good alternative to triangular osteosynthesis while simultaneously reducing the undesired consequences of lumbopelvic fixation. As a biomechanical study, however, the clinical benefits remain to be proven.

\section{Conflict of Interest}

No potential conflict of interest relevant to this article was reported.

\section{References}

1. Routt ML Jr, Kregor PJ, Simonian PT, Mayo KA. Early results of percutaneous iliosacral screws placed with the patient in the supine position. J Orthop Trauma 1995;9:207-14.

2. Ebraheim NA, Coombs R, Hoeflinger MJ, Zeman C, Jackson WT. Anatomical and radiological considerations in compressive bar technique for posterior pelvic disruptions. J Orthop Trauma 1991;5:434-8.

3. Albert MJ, Miller ME, MacNaughton M, Hutton WC. Posterior pelvic fixation using a transiliac $4.5-\mathrm{mm}$ reconstruction plate: a clinical and biomechanical study. J Orthop Trauma 1993;7:226-32.

4. Pohlemann T, Angst M, Schneider E, Ganz R, Tscherne H. Fixation of transforaminal sacrum fractures: a biomechanical study. J Orthop Trauma 1993;7:107-17.

5. Keating JF, Werier J, Blachut P, Broekhuyse H, Meek RN, O'Brien PJ. Early fixation of the vertically unstable pelvis: the role of iliosacral screw fixation of the posterior lesion. J Orthop Trauma 1999;13:107-13.

6. Griffin DR, Starr AJ, Reinert CM, Jones AL, Whitlock S. Vertically unstable pelvic fractures fixed with percutaneous iliosacral screws: does posterior injury pattern predict fixation failure? J Orthop Trauma 2003;17:399-405.

7. Schildhauer TA, Ledoux WR, Chapman JR, Henley MB, Tencer AF, Routt ML Jr. Triangular osteosynthesis and iliosacral screw fixation for unstable sacral fractures: a cadaveric and biomechanical evaluation under cyclic loads. J Orthop Trauma 2003;17:22-31.

8. Papakostidis C, Kanakaris NK, Kontakis G, Giannoudis PV. Pelvic ring disruptions: treatment modalities and analysis of outcomes. Int Orthop 2009;33:329-38. 
9. Kach K, Trentz O. Distraction spondylodesis of the sacrum in "vertical shear lesions" of the pelvis. Unfallchirurg 1994;97:28-38.

10. Denis F, Davis S, Comfort T. Sacral fractures: an important problem: retrospective analysis of 236 cases. Clin Orthop Relat Res 1988;227:67-81.

11. Schildhauer TA, Josten Ch, Muhr G. Triangular osteosynthesis of vertically unstable sacrum fractures: a new concept allowing early weight-bearing. J Orthop Trauma 2006;20(1 Suppl):S44-51.

12. Mouhsine E, Wettstein M, Schizas C, et al. Modified triangular posterior osteosynthesis of unstable sacrum fracture. Eur Spine J 2006;15:857-63.

13. Sagi HC. Technical aspects and recommended treatment algorithms in triangular osteosynthesis and spinopelvic fixation for vertical shear transforaminal sacral fractures. J Orthop Trauma 2009;23:354-60.

14. Sagi HC, Militano U, Caron T, Lindvall E. A comprehensive analysis with minimum 1-year follow-up of vertically unstable transforaminal sacral fractures treated with triangular osteosynthesis. J Orthop Trauma 2009;23:313-9.

15. Hao Z, Wan C, Gao X, Ji T. The effect of boundary condition on the biomechanics of a human pelvic joint under an axial compressive load: a threedimensional finite element model. J Biomech Eng 2011;133:101006.

16. Miller AN, Routt ML Jr. Variations in sacral morphology and implications for iliosacral screw fixation. J Am Acad Orthop Surg 2012;20:8-16.
17. Templeman D, Schmidt A, Freese J, Weisman I. Proximity of iliosacral screws to neurovascular structures after internal fixation. Clin Orthop Relat Res 1996;(329):194-8.

18. Ebraheim NA, Xu R, Biyani A, Nadaud MC. Morphologic considerations of the first sacral pedicle for iliosacral screw placement. Spine (Phila Pa 1976) 1997;22:841-6.

19. Vaccaro AR, Kim DH, Brodke DS, et al. Diagnosis and management of sacral spine fractures. Instr Course Lect 2004;53:375-85.

20. Hu X, Pei F, Wang G, He J, Kong Q, Tu C. Application triangular osteosynthesis for vertical unstable sacral fractures. Eur Spine J 2013;22:503-9.

21. Bents RT, France JC, Glover JM, Kaylor KL. Traumatic spondylopelvic dissociation: a case report and literature review. Spine (Phila Pa 1976) 1996;21:18149.

22. Sullivan MP, Smith HE, Schuster JM, Donegan D, Mehta S, Ahn J. Spondylopelvic dissociation. Orthop Clin North Am 2014;45:65-75.

23. Park YS, Baek SW, Kim HS, Park KC. Management of sacral fractures associated with spinal or pelvic ring injury. J Trauma Acute Care Surg 2012;73:239-42.

24. Chong AC, Miller F, Buxton M, Friis EA. Fracture toughness and fatigue crack propagation rate of short fiber reinforced epoxy composites for analogue cortical bone. J Biomech Eng 2007;129:487-93.

25. Heiner AD. Structural properties of fourth-generation composite femurs and tibias. J Biomech 2008;41:3282-4. 